\title{
Biochemical munchausen's - a 'baffling” case of recurrent hypokalemia and lactic acidosis - secondary to surreptitious salbutamol abuse in a 36-year-old female
}

\author{
Cameron Chen ${ }^{1}$, Udayan Ray ${ }^{1}$, Glenn Jacobson ${ }^{2}$, Michael Smillie ${ }^{1}$, Neville Jordan ${ }^{1}$, Richard $\mathrm{Yu}^{* 3}$ \\ ${ }^{1}$ Department of Biochemistry, Royal Hobart Hospital, Tasmania, Australia \\ ${ }^{2}$ School of Pharmacy, University of Tasmania, Australia \\ ${ }^{3}$ Department of Nephrology and General Medicine, Royal Hobart Hospital, Australia
}

Received: April 20, 2016

DOI: $10.5430 /$ crim.v3n3p17
Accepted: May 30, 2016

Online Published: June 5, 2016

URL: http://dx.doi.org/10.5430/crim.v3n3p17

\begin{abstract}
Factitious medical disorders with the well-known eponym of Munchausen's syndrome represent a challenge in both diagnosis and management for the clinician. Here, we report a case of "Biochemical Munchausen's" in a 36-year-old female, characterised by recurrent and multiple presentations with symptomatic hypokalaemia, supraventricular tachycardia and lactic acidosis that required the use of biochemical laboratory expertise and liquid chromatography/mass spectrophotometry to unravel and break the cycle of costly investigations and hopefully contain the risk of potential harm. We also highlight the evolution of various stages of generating factitious illness in this 36-year-old female with the crucial breakthrough being made only by proof of the biochemical exposure through the close collaboration of biochemist and clinicians.
\end{abstract}

Key Words: Hypokalemia, Lactic acidosis, Munchausen's, Salbutamol, Tachycardia

\section{INTRODUCTION}

Factitious medical disorders with the eponym of Munchausen's syndrome represents a challenge in both diagnosis and management for the clinician. This is because the spectrum of this disorder goes against the fundamental assumptions of the therapeutic relationship that lies at the heart of medicine. While the eponym, named after the fantastical 18th century German raconteur Baron Munchausen (17201797) was first used by Richard Asher in a case series in $1951,{ }^{[1]}$ the condition is likely to have existed for almost as long as the patient physician relationship has been around.
Numerous presentations and variations are reported sometimes with incredible degrees of ingenuity in engineering factitious illness demonstrated by the individual patient.

Here, we report a case of "Biochemical Munchausen's" in a 36-year-old female, characterised by recurrent and multiple presentations with symptomatic hypokalaemia, supraventricular tachycardia and lactic acidosis that required the use of laboratory expertise and liquid chromatography/mass spectrophotometry to unravel and break the cycle of costly investigations and hopefully contain the risk of potential harm. Recurrent and surreptitious abuse of salbutamol was ultimately

\footnotetext{
*Correspondence: Richard Yu; Email: richard.yu@ths.tas.gov.au; Address: Department of Nephrology and General Medicine, Royal Hobart Hospital, Australia.
} 
revealed to be the mechanism for generating our patient's seemingly baffling biochemical findings.

We also highlight the evolution of various stages of generating factitious illness in this 36-year-old female with the crucial breakthrough being made only by proof of the biochemical exposure through the close collaboration of biochemist and clinicians.

\section{CASE REPORT}

The patient was a 36-year-old woman who was previously well. Her prior medical history with our institution included a documentation of asthma, perianal and breast abscesses, colonic polyps and hidradenitis suppurativa. She was a married housewife with four children and a current smoker. Her only medications were salbutamol as required for her asthma (which she denied using) and paracetamol.

She first presented to the Emergency Department of our hospital in July 2007 with chest pain, nausea and diaphoresis. ECG revealed a supraventricular tachycardia (SVT) with a rate of $140 \mathrm{bpm}$ and eventual spontaneous resolution to normal sinus rhythm. She was referred to the Cardiology team where she was prescribed at various stages flecainide, atenolol and verapamil for paroxysmal SVT all without longterm success in reducing the frequency of her presentations.
She was euthyroid with a normal 2D Echocardiography.

Four months after her initial presentation with symptomatic paroxysmal tachycardia she had had ten presentations to our Emergency Department with stereotypical self-resolving symptoms of symptomatic SVT. Given the disruption to her family life and symptomatic nature of her tachycardia, she was referred at his point by the cardiology team for Electrophysiological Study (EPS) at an interstate quarternary centre with the provisional diagnosis of refractory paroxysmal SVTmost likely atrial tachycardia

Her EPS performed five months after her first presentation was essentially normal with no inducible focus of atrial tachycardia and a diagnosis of inappropriate sinus tachycardia was made. She was recommenced by our cardiology team on Verapamil and a beta-blocker for management of what was felt to be a highly symptomatic inappropriate sinus tachycardia. Pharmacotherapy did not however reduce the frequency or the reported symptomatic nature of her tachycardia presentations to emergency.

By the time of her return from her interstate EPS assessment, her blood results during each symptomatic tachycardic presentation was increasingly characterised by hypokalemia- to levels as low as $2.6 \mathrm{mmol} / \mathrm{L}$ and variable degrees of lactic acidosis (up to 4-6 mmol/L) and at times hyperglycemia.

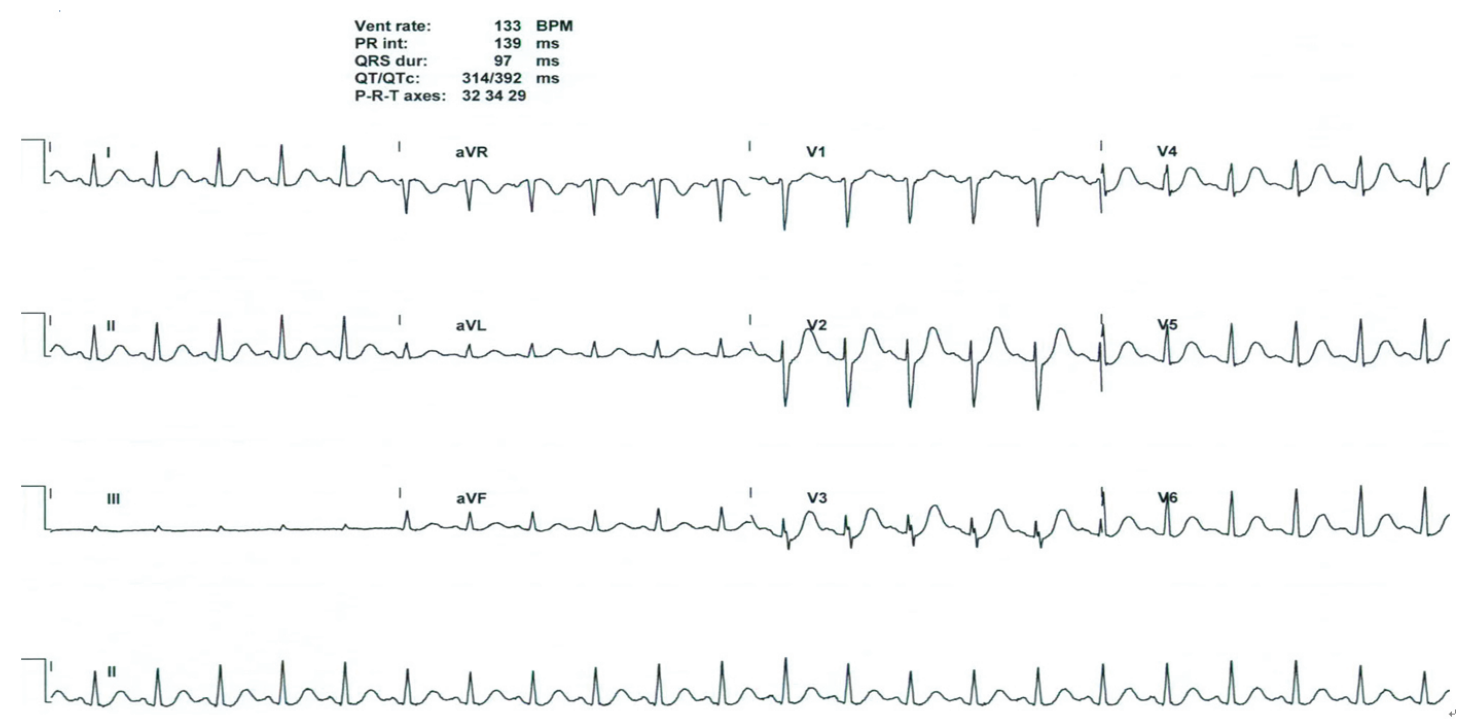

Figure 1. ECG during a typical presentations with symptomatic but self resolving tachycardia and frequent associated biochemical triad of hypoklemia, Lactic acidosis and hyperglycemia

Her presentations to the Emergency Department with a constellation of stereotypical features occurred at approximately weekly intervals for the following two years, with the biochemical findings of hypokalaemia, lactic acidosis, transient hyperglycaemia alongside sinus tachycardia and occasionally
SVT.

On each occasion, she reported symptoms of palpitations, muscle weakness and cramping. She was treated with intravenous potassium replacement and intravenous fluids- with eventual resolution of her symptoms and all ECG and bio- 
chemical findings after 4-6 hours of treatment and observation.

Table 1. Biochemistry from venous gases in a series of three typical presentations (out of many) is shown below with our laboratory Reference ranges in parenthesis. Note the significantly elevated lactate (up to $7.5 \mathrm{mmol} / \mathrm{L}$ ), profound hypokalemia (down to $2.6 \mathrm{mmol} / \mathrm{L}$ ) and mild metabolic acidosis.

\begin{tabular}{lllll}
\hline Items & \multicolumn{2}{l}{ Value } & & Reference Range \\
\hline $\mathrm{pH}$ & 7.41 & 7.320 & 7.39 & $(7.35-7.45) \mathrm{mmHg}$ \\
$\mathrm{pCO}_{2}$ & 28 & 37 & 34 & $(35-45) \mathrm{mmHg}$ \\
$\mathrm{pO}_{2}$ & 48 & 58 & 26 & $(80-100) \mathrm{mmHg}$ \\
$\mathrm{cHCO}_{3}$ & 18 & 18 & 20 & $(22-30) \mathrm{mmol} / \mathrm{L}$ \\
Base Excess & -5 & -6 & -4 & $(-3 / 3) \mathrm{mmol} / \mathrm{L}$ \\
Potassium & 2.7 & 2.6 & 3.1 & $(3.5-5.0) \mathrm{mmol} / \mathrm{L}$ \\
Chloride & 108 & 108 & 105 & $(95-110) \mathrm{mmol} / \mathrm{L}$ \\
Ca Ion pH 7.4 & 1.16 & 1.12 & 1.16 & $(1.14-1.29) \mathrm{mmol} / \mathrm{L}$ \\
Glucose & 9.6 & 10.2 & 7.5 & $(2.5-7.8) \mathrm{mmol} / \mathrm{L}$ \\
Lactate & 5.7 & 7.1 & 7.2 & $(0.6-2.4) \mathrm{mmol} / \mathrm{L}$ \\
\hline
\end{tabular}

The hypokalaemia was not accompanied by hypertension or metabolic alkalosis, suggesting that Cushing's syndrome or mineralocorticoid excess was not a cause. A diuretic and laxative screen performed was negative. She co-operated fully with all medical investigations and was accepting of the uncertainty of her diagnosis and the joint involvement of multiple teams. She was questioned on potential surreptitious agents including in particular, beta-agonists, but strenuously denied any such potential exposure and, perhaps understandably reported feeling "hurt" by this line of questioning by clinical staff.

In October 2007, the patient was reviewed by the Nephrology team. Regarding her transient but profound hypokalemia. The possibility of Bartter's/Gitelman's syndrome was raised but most of the biochemical and clinical features including the lack of metabolic alkalosis and the salience of lactic acidosis refuted this diagnosis. Similarly, Renal Tubular acidosis (RTA) was entertained but refuted by the biochemical finding of an elevated lactate as the driver for her transient episodes of mild acidemia. The presence of lactic acidosis on each occasion raised the possibility of a poorly differentiated mitochondrial cytopathy. Increasingly, the spectrums of these disorders are being recognised including various forms of renal involvement. A renal biopsy was carried out in July 2008 (16 months after her initial presentations) to ex- amine for any morphological abnormalities of mitochondria on electron microscopy. Electron Microscopy demonstrated non-specific changes in renal tissue mitochondria but no specific evidence of a mitochondrial cytopathy with renal involvement.

In the setting of her recurrent episodes of symptomatic hypokalemia, a channelopathy including the possibility of Hypokalemic Periodic Paralysis was entertained but refuted by the other clinical features of unexplained lactic acidosis. Neurology review for her constellation of baffling symptoms and biochemical abnormalities, with a particular emphasis on the question of an unrecognised mitochondrial disorder led to further investigations. MRI showed non-specific white matter abnormalities and CSF in search of elevated lactate, pyruvate and pleocytosis that can be associated with MELAS (Mitochondrial Myopathy, Encephalopathy, Lactic Acidosis and Stroke syndrome) was entirely unremarkable. A muscle biopsy was performed in February 2008 in Melbourne which revealed no obvious mitochondrial abnormality and no direct evidence to support a mitochondrial disorder. Testing for the MELAS 3243A > G gene mutation associated with MELAS was also negative.

By July 2008 (16 months after her initial presentation), recurrent parenteral potassium during treatment for her multiple symptomatic hypokalemic presentations meant that she was running out of intravenous access to aid with potassium replacement during each symptomatic presentation. An infusaport was needed to facilitate the management plan of her intravenous fluid and potassium replacement, which was instituted on each presentation whilst her symptoms and the biochemical triad of hypokalemia, lactic acidosis and transient hyperglycemia subsided.

In July 2008, soon after infusaport insertion she presented with fevers and a positive blood culture. Blood cultures showed polymicrobial growth with organisms suggestive of contamination via her infusaport. She was reviewed by the Infectious Disease Team and underwent Trans-oesophageal Echocardiography, CT imaging and Gallium and White Cell scan over the course of these events to exclude a deep seated infection- all of which was negative. The possibility of deliberate contamination of her vascular device was raised by our Infectious Disease team given the unusual recurrent polymicrobial nature of blood culture findings in the absence of any focal source.

However, no direct proof of deliberate contamination of her infusaport or deliberate chemical exposure could be demonstrated and she strenuously denied any such possibility and once again reported feeling hurt by these discussions. 
In the ensuing 5 months, she experienced 5 distinct episodes of illness related to positive blood cultures- usually of a polymicrobial nature including Strep Sanguinous and Strep Oralis. Candida Albicans was isolated in one blood culture resulting in infusaport removal and later changes of infusaport.

These bacteraemic and fungemic presentations were now interspersed with the previous features of tachycardia (sinus or SVT) and symptomatic biochemical abnormalities described above.

The possibility of factitious illness was again revisited but with no strong corroborating evidence to refute or support this hypothesis. The only likely agent felt to be available to her to generate these symptoms was a beta-agonist but a check with her local pharmacy failed to reveal any recent prescriptions for this class of agents. Biochemical assay for salbutamol and other beta-agonists is not routinely available in hospital laboratories as ordinarily, such an assay would have no clinical utility.

On discussion through our hospital biochemists with the University Faculty of Pharmacy associated with our tertiary hospital, access was obtained to a salbutamol assay.

Almost two years after initial presentation, and following more than 60 hospital admissions in that time, a serum sample was sent to the Faculty of Pharmacy at the University of Tasmania during one of her stereotypical presentations. Salbutamol level was measured by liquid chromatography/mass spectrometry to be $5 \mathrm{ng} / \mathrm{ml}$, which is more than double the expected concentration after therapeutic use for most patients following an exacerbation of asthma. (reference range for peak plasma concentration after $0.04-0.1 \mathrm{mg}$ inhaler dose $=0.6-1.4 \mathrm{ng} / \mathrm{ml}$ ).

After the cause of the patient's symptoms was ascertained, the results of our assay were discussed with her. She strenuously denied any such exposure and responded with anger to any suggestion of factitious illness. She was counselled and offered linkage to see our Liaison Psychiatry service. Unfortunately, as is often reported to be the case in patients with Munchausen's type pathology, she disengaged from our medical service entirely after being confronted with findings that strongly pointed to surreptitious beta-agonist use as the cause of her symptoms. She was lost to follow up and subsequent attempts by our hospital to contact her were unsuccessful. Her General Practitioner was informed of the results of these investigations and a written plan instituted to help contain the risks from prescription patterns that might become the focus of factitious illness. Unfortunately, to date she has not re-engaged with the medical system to seek counselling or help.

\section{Discussion}

Hypokalaemia is frequently seen in the hospital setting. Common causes for hypokalaemia include alkalosis, when there is a shift of potassium into the cell membrane. Metabolic alkalosis may be due to excess mineralocorticoid or glucocorticoid, medications (namely, diuretics and penicillins), and vomiting or nasogastric suction.

Bartter's and Gitelman's syndromes are congenital disorders of the kidney tubules, affecting the thick ascending loop of Henle and the distal convoluted tubule, respectively. Five different genes for Bartter's syndrome have been recognised, each resulting in dysfunction of some aspect of regulated sodium, potassium and chloride transport in the thick ascending loop. Gitelman's syndrome is due to a mutation affecting the thiazide-sensitive sodium-chloride transporter of the distal convoluted tubule. However it would be unusual for these congenital conditions to first appear in the fourth decade of life, nor do the other biochemical features in this case in any way support this as a basis for her hypokalemia.

The combination of hypokalaemia and metabolic acidosis is clinically seen much more rarely. Distal and Proximal renal tubular acidosis (or RTA Type I- Distal and Type II- Proximal respectively) is characterised biochemically by a combination of hypokalaemia and normal anion gap metabolic acidosis. However, in RTA, the metabolic acidosis is never characterised by lactic acidosis.

Diuretic abuse may mimic Bartter's and Gitelman's syndrome, with the former being mimicked by the excessive administration of loop diuretics and the latter with thiazide diuretics. This is because loop diuretics such as frusemide target the Na-K-2Cl symporter in the thick ascending limb of the loop of Henle which is defective in Type 1 (neonatal) Bartter's syndrome, and thiazide diuretics target the distal convoluted tubule's sodium-chloride co-transporter that is affected in patients with Gitleman's. In this particular case, the patient denied taking either type of diuretic. Moreover, neither of these conditions would result in a raised serum lactate.

Salbutamol is a selective beta- 2 agonist used for symptomatic treatment for asthma. Hypokalaemia is a well-documented side effect. Indeed, beta-agonists are frequently recommended in the short term management of hyperkalemia in numerous therapeutic guidelines. Previous literature has documented significant rates of hypokalaemia after administration of salbutamol in children. One case report has also stated that overdose of salmeterol may cause a lactic acidosis in addition to hypokalaemia. ${ }^{[6]}$ 
Munchausen's syndrome is an uncommon factitious disorder, with patients often lying, intentionally reporting symptoms and frequently being hospitalised with the intent of playing a "sick" role. ${ }^{[2,3]}$ The condition appears to be perpetuated by psychological rather than any quantifiable material gain resulting from the "sick" role and requires early recognition, exclusion of organicity and an empathetic approach to treatment ${ }^{[3]}$.

There exists little previous literature regarding factitious drug toxicity leading to electrolyte disturbances. One case was documented of a 26-year-old woman who presented multiple times to the emergency department with supraventricular tachycardia and transient hypokalaemia over a 3-year-period from salbutamol (albuterol) misuse. ${ }^{[4]}$ This patient was also eventually lost to follow-up. There have also been reported cases of surreptitious use of loop and thiazide diuretics leading to the mistaken initial diagnosis of Bartter's syndrome ${ }^{[5]}$ and Gitelman syndrome respectively. It's likely that many cases of Munchausen's type pathology go unreported and at times undetected.

Our case is interesting on a few fronts. Firstly, it illustrates what we believe in retrospect to be a gradual evolution of her factitious illness, through three distinct clinical phases. We hypothesise that the evolution through each phase was coloured by information she may have gathered on her interactions with clinicians involved in her care. These 3 phases can be broadly characterised as follows:

(1) Arrhythmogenic Munchausen's characterised by repeated self-dosing with salbutamol, sufficient to generate a sinus tachycardia or SVT- but insufficient to generate significant biochemical abnormalities which were later seen.

(2) Biochemical Munchausen's, characterised by higher surreptitious dosing of salbutamol sufficient to generate not only the earlier tachycardic phenomenon, but also a biochemical triad of hypokalemia, lactic acidosis and transient hyperglycemia.

(3) Lastly, the creation of an infusaport to manage her baffling episodes of symptomatic hypokalemia led to a third phase of factitious illness that one might term Bacteremic Munchausen's characterised by what we suspect is likely to have been deliberate self- inoculation of vascular access devices resulting in polymicrobial bacteremia with organisms found in oral flora.

We hypothesise that our patient had begun her interactions with surreptitious salbutamol use but with feedback through her numerous interactions with treating teams regarding the clinical problems encountered, including information about the biochemical abnormalities encountered, she may have evolved in her dosing and subsequent patterns of risk behaviour. As such this represents a unique case of Munchausen's in which there is an evolution of surreptitious illness through new clinical information gathered by the patients through her interactions with the hospital and multiple treating teams.

Ultimately, proof via mass spectrometry/liquid chromatography of the biochemical agent responsible for her presentations allowed us the opportunity to frankly discuss these issues with her and offer help through our Liaison Psychiatry service. Unfortunately, as in most cases of Munchausen's, reported in the literature, these patients often deny factitious illness despite overwhelming proof and all too frequently disengage from the medical system, sometimes to emerge later.

The multitude of investigations undertaken for this patient is believed to have cost the health system hundreds of thousands of dollars. While factitious disorder is rare, when unrecognised it has the potential to be very costly to health systems and potentially exposes the patient to avoidable iatrogenic harm through the process of investigations or unwarranted treatment. In cases of repeated electrolyte abnormality of an unknown aetiology, consideration must be given with respect to toxicity from external agents. Investigation of such a possibility may potentially avoid wastage of a substantial amount of public funds, although factitious disorder may be difficult to recognise in the clinical setting.

\section{REFERENCES}

[1] Richard A. Munchausen's Syndrome. The Lancet. 1951; 1: (6650): 339-41. http://dx.doi.org/10.1016/S0140-673 6(51) $92313-6$

[2] Leikin JB, Linowiecki KA, Soglin DF, et al. Hypokalaemia after pediatric albuterol overdose: a case series. Am J Emerg Med. 1994; 12(1): 64-6. http://dx.doi.org/10.1016/0735-6757 (94)90202-X

[3] Prakash J, Das RC, Srivastava K, et al. Munchausen syndrome: Playing Sick or Sick player. Ind Psychiatry J. 2014; 23(1): 68-70. PMid:25535450 http://dx.doi.org/10.4103/0972-6748.14 4975

[4] Wills BK, Kwan C, Bailey M, et al. Recalcitrant supraventricular tachycardia: Occult albuterol toxicity due to a factitious disorder. J Emerg Med. 2015; 49(4): 436-8. PMid:26162766 http: //dx.doi.org/10.1016/j.jemermed.2015.05.007

[5] Sekine K, Kojima I, Fujita T, et al. Factitious Bartter's Syndrome induced by Surreptitious intake of Furosemide. Endocrinol Japan. 1982; 29(5): 653-7. http://dx.doi.org/10.1507/endocrj19 54.29 .653

[6] Manara A, Hantson P, Vanpee D, et al. Lactic acidosis following intentional overdose by inhalation of salmeterol and fluticasone. Canadian J of Emergency Medicine. 2012; 14(6): 378-81. 